\title{
Controller for Integrator Processes
}

\author{
B. S. Patil \\ Instrumentation Department, \\ AISSMS'S Polytechnic, \\ Near RTO office, Kennedy \\ Road, Pune-01
}

\author{
L. M. Waghmare, $\mathrm{PhD}$ \\ Director, Shri Guru Gobind \\ Singhji, Institute of Engineering \\ \& Technology, Vishnupuri, \\ Nanded - 431606, India
}

\author{
M. D. Uplane, PhD \\ HOD, Professor, \\ Instrumentation Science \\ Department, Savitribai Phule \\ Pune University, Pune
}

\begin{abstract}
A design method for PID controllers base on internal model control (IMC) principles, direct synthesis method (DS), stability analysis (SA) system for pure integrating process with time delay is proposed. Analytical expressions for PID controllers are derived for several common types of process models, including first order and second-order plus time delay models and an integrator plus time delay model. Here in this paper, a simple manager design rule and tuning procedure for unstable processes with delay time is discuss. Simulation examples are included to show the effectiveness of the proposed method.
\end{abstract}

\section{Keywords}

PID controller, tuning, unstable processes, internal model control (IMC), direct synthesis method (DS), stability analysis (SA).

\section{INTRODUCTION}

The ubiquitous PID controller has continued to be the most usually used process control technique for many decades. Although advanced control techniques such as model predictive control can supply significant improvements, a PID controller that is properly designed and tune has proved to be suitable for the vast majority of industrial control loops. The enormous literature on PID controllers includes a wide selection of design and tuning methods based on different performance criteria Incorporating systems with time suspension are create in the model of liquid level system, liquid storage tank, boilers, batch chemical reactors and the bottom level manage of a distillation column[1]. Here we investigate optimal PID control of a double integrating plus delay process. What makes the double integrate process individual, is that derivative action is actually necessary for stabilization.

DS design methods are typically based on specification of the desired closed-loop transfer functions for set-point changes. Consequently, the resulting DS controllers tend to execute well for set-point changes, but the disturbance response might not be satisfactory. The IMC-PID controller provides good set point tracking but very sluggish disturbance responses for processes with a small time-delay/time-constant ratio. Fuentes and Luyben [2] have reported that the composition control loop of a high purity distillation column

has a large time constant and therefore, the response resemble that of a pure integrator plus dead time model. An isothermal continuous copolymerization reactor can be modeled as an integrating system with dead time [3]. The representation contain only two parameter ( $k$ and $L p$ ) and the model is very easy for identification. The model is able to sufficiently represent the dynamics of many systems over the frequency range of interest for the PID controller design.

\section{LITERATURE REVIEW}

Many researchers have published their own work in design of. PID controller. Some of the important publications reported are as follows:

Dan then. and Dale E. Seborg, "P1/PIO. Controller Design Based on Direct Synthesis and Disturbance Rejection" [1]

Authors have given a design method for PM controller based on the direct synthesis approach and specification of :the desired closed-loop transfer function for disturbance os proposed. Analytical expression for PID controller are derived for several common types of process models, including first order and second-order plus time delay model. Although the controller are designed for disturbance rejection, the set-point responses are usually satisfactory and can be tuned independently via a set-point weighting factor. Nine simulation example demonstrate that the proposed design method results in very good control for a wide variety of processes including those with integrating and /or minimum phase characteristic rejection than the standard direct synthesis and internal model control methods when the controller are tuned to have the same degree of robustness.

G.M. Malwatkar, A.A. Khandejkar and archana, K. Choudhari, "Tuningh PI controller for integrating non minimum phase plus delay time processes" [2]

Authors have given a direct synthesis method for disturbance rejection (DS-d) is employed to derive a PI controller for integrating process with non minimum phase and delay. The delay is approximated by first-order Taylor Series approximation and the phase and integrator. The model order reduction is based on frequency response technique. The tuning parameter A for DS-d is calculated by using minimum an integral absolute error (IAR) Using golden section search method. Simulation examples are included to show effectiveness of the proposed controller. The technique proposed can perform well for load/disturbance change.

E. Poulin, A. Pomerleau, "PID tuning for integrating and unstable processes" [3]

Authors have given a systematic PI and PID tuning method is developed for integrating and unstable processes. The method based on a maximum peak-resonance specification, leads to simple tuning parameter expressions and is graphically supported by the Nichols chart. The common characteristic of

integrating and unstable processes is that their open-loop frequency response, with the controller in cascade, has a phase maximum. The controller parameters are adjusted such that this maximum is located on the right-most point of the ellipse, corresponding to the maximum peak resonance on the Nichols chart. For these types of processes, making the openloop frequency-response curve tangent to a specified ellipse is an efficient method of controlling the overshoot, the stability 
and the dynamic of the system. Charts that give the optimal peak resonance according to the ITAE criterion, are given. The typical obtained with this method are presented. for typical design examples. A comparison of the performance of the proposed design setting with those given by earlier workers for unstable processes is presented.

L. Wagh, W.R. Clutt, "tuning PID controller for integrating processes" [4]

Authors have given the frequency domain PID controller design method, first proposed by the authors in an earlier paper, by presenting a comprehensive treatment of integrating process. The general class of integrating process is divided

into two types, based on the sign of the second coefficient of the Taylor series expansion applied to the stable part of the process transfer function. In both case, the closed -loop performance is specified iii terms of the desired control-signal trajectory scaled with respect to the magnitude of this coefficient. In addition, explicit PID tuning rules are given, for integrating plus delay processes along with their associated gain and phase margins and allowable time delay variations.

L. Ou, W. Zhang and D. Gu, "Sets of - stabilizing PID controller for second-order integrating processes with time delay" [5]

Authors have given procedures for determining the complete sets of proportional integral derivative (PID) controller are presented for three classes of second-order integrating processes with time delay. The necessary condition for the ND controller to stabilize second-order integrating processes with time delay is first derived in the case where frequency, w equals zero of infinity. On the basis of the extended Hermite Biehler theorem, analytical formula are provided to determine the admissible ranges of the proportional gain $(\mathrm{kp})$, internal gain(ki), and the derivative gain(M) respectively, and the complete set of stabilizing PID controllers is found by sweeping over the entire range of allowable $\mathrm{kp}$ value. The stability analysis on the basis of the extended Hermite-Biehler theorem shot that the stability boundary in the controller parameter space depends only on the frequency in the defined small range. In view of this, the set of stabilising PM controller are also determined by sweeping over the admissible ranges of $\mathrm{kci}$ and $\mathrm{ki}$, respectively. As a result, for a given second-order integrating process with time delay, the complete set of stabilising PID controller can be determined by sweeping any one of the PID parameters over the corresponding admissible range.

Eelena Grassi, Kostas S. Tsakailis, Sachi Dash, Sujit V. Gaikwad, Ward MacArthur, and Gunter Stein, "Integrating system identification and PID Controller Tuning by Frequency Loop- Shaping"[6]

Authors have given system antic design methodology for proportional integral derivative (PID) controllers. Starting from data sets, a model of the system and its uncertainty bounds are obtained. The parameter of the controller are tuned by a convex the actual loop transfer function and a target in a L2/L1 sense. The target selection is guided by the identified model and it uncertainty. The problem of disjoint data sets and/or different models for same system is also addressed. The method has proved successful in numerous practical cases showing both expediency in controller design and implementation and improved performance over existing controllers.

\section{METHODS IN PROPOSED SYSTEM}

The present work is intended to design PID controllers for pure integrating systems with time delay using three methods (i) IMC method (ii) direct synthesis method and (iii) stability analysis method.

\section{IMC Method}

A well-known control system design strategy, internal model control (IMC) was developed by Morari and co-workers20 and is closely related to the direct synthesis approach. Like the DS method, the IMC method is based on an assumed process model and relates the controller settings to the model parameters in a straightforward manner. The IMC approach has the advantages that it makes the consideration of model uncertainty and the making of tradeoffs between control system performance and robustness easier.

The process transfer function is given by

$$
G_{p}=\frac{k_{p} e^{-L s}}{s}
$$

Using Pade's approximation for time delay, Eq (1) is rewritten as

$$
G_{p}=\frac{k_{p}(1-0.5 L s)}{s(1+0.5 L s)}
$$

IMC controller for the above system consists of two parts. First part is the inverse of the stable portion of the process and second part is IMC filter. The numerator order of the IMC filter is equal to the number of unstable poles.

\section{Direct Synthesis method}

In general, both the direct synthesis and IMC methods do not necessarily result in PI/PID controllers. However, by choosing the appropriate desired closed-loop response and using either a Pade' approximation or a power-series approximation for the time delay, PI/PID controllers can be derived for process models that are commonly used in industrial applications.

The process transfer function is given by $\mathrm{Eq}$ (1). The controller transfer function istaken as

$$
\begin{aligned}
& G_{C}=k_{c}\left(1+\frac{1}{\tau_{I} s}+\tau_{D} s\right) \frac{1}{\left(1+\tau_{f} s\right)} \\
& \text { Where } \tau_{f}=\beta \tau_{D}
\end{aligned}
$$

\section{Stability analysis method}

Generally we will deal with a semi discrete model: discrete in space and continuous in time. In the time domain the model is given by ordinary differential equations (ODE) in time.

Amplification Methods. Also called von Neumann stability analysis. Based on decomposition of motion into normal modes, often using Fourier analysis, and superposition. The analysis looks at the growth or decay of perturbations from one step to the next, and can be implemented using standard linear algebra procedures. It is local in nature, but so is the concept of stability. Amore severe restriction is that it strictly applies only to linear systems. Despite this limitation it is frequently applied to nonlinear systems through linearization. Energy Methods. Also known, notably in control theory, as 
Lyapunov methods. These look at the variation of certain function (or functional) measures of the motion amplitude. Often these are related to energy measures of various kinds, hence the name. Energy methods are not restricted to linear systems, but require the construction of suitable measures, and this has to be done case by case.

The process transfer function for pure integrator with time delay is given by equation Eq (1). The phase angle criterion for pure integrator with time delay is given by

$$
\phi(\omega)=-\frac{\pi}{2}-L \omega
$$

\section{Kharitonov's theorem:}

The stability regions of the model parameters for the PID controller designed are calculated by Kharitonov's theorem considering uncertainty in one parameter at a time. Kharitonov's theorem is a result used in control theory to assess the stability of a dynamical system when the physical parameters of the system are not known precisely. When the coefficients of the characteristic polynomial are known, the Routh-Hurwitz stability criterion can be used to check if the system is stable (i.e. if all roots have negative real parts). Kharitonov's theorem can be used in the case where the coefficients are only known to be within specified ranges. It provides a test of stability for a so-called interval polynomial, while Routh-Hurwitz is concerned with an ordinary polynomial.

An interval polynomial is the family of all polynomials

$$
p(s)=a_{0}+a_{1} s^{1}+a_{2} s^{2}+\ldots+a_{n} s^{n}
$$

where each coefficient can take any value in the specified intervals $\boldsymbol{u}_{\boldsymbol{i}} \leq \boldsymbol{a}_{\boldsymbol{i}} \leq \boldsymbol{u}_{\boldsymbol{i}}$. It is also assumed that the leading coefficient cannot be zero: $\mathbf{0} \notin\left[\mathrm{s}^{s s^{2}} \mathrm{~s}^{2 s}\right]$.

An interval polynomial is stable (i.e. all members of the family are stable) if and only if the four so-called Kharitonov polynomials are stable.

$$
\begin{aligned}
& k_{1}(s)=l_{0}+l_{1} s^{1}+u_{2} s^{2}+u_{3} s^{3}+l_{4} s^{4}+l_{5} s^{5}+\cdots \\
& k_{2}(s)=u_{0}+u_{1} s^{1}+l_{2} s^{2}+l_{3} s^{3}+u_{4} s^{4}+u_{5} s^{5}+\cdots \\
& k_{3}(s)=l_{0}+u_{1} s^{1}+u_{2} s^{2}+l_{3} s^{3}+l_{4} s^{4}+u_{5} s^{5}+\cdots \\
& k_{4}(s)=u_{0}+l_{1} s^{1}+l_{2} s^{2}+u_{3} s^{3}+u_{4} s^{4}+l_{5} s^{5}+\cdots
\end{aligned}
$$

is somewhat surprising about Kharitonov's result is that although in principle we are testing an infinite number of polynomials for stability, in fact we need to test only four. This we can do using Routh-Hurwitz or any other method. So it only takes four times more work to be informed about the stability of an interval polynomial than it takes to test one ordinary polynomial for stability.

Kharitonov's theorem is useful in the field of robust control, which seeks to design systems that will work well despite uncertainties in component behavior due to measurement errors, changes in operating conditions, equipment wear and so on.

\section{CONCLUSION AND FUTURE WORK}

Three methods of designing PID controllers for pure integrating system with time delay are proposed based on IMC method, stability analysis method and direct synthesis method. The performance of the proposed controllers is better than the recently reported methods. Stability region for various model parameters considering uncertainty in one parameter at a time is obtained using theorem and compared with that of the literature reported methods. The stability region for all the model parameters is comparable with that of the literature reported methods. The advantage of these methods is that the controller is PID and simple conventional feed back control structure is used.

\section{REFERENCES}

[1] Chien, L. and Fruehauf, P. S. 1990. ConsiderIMC tuning to improve performance.ChemEngProgr, 10: 33-41.

[2] Fuentes, C. and Luyben, W. L. 1983.Control of high purity distillation columns.IndEngChem Process Des Dev,22: 362 .

[3] Srividya, R. and Chidambaram, M. 1997.On line controller tuning for integratorplus delay processes. Process ContrQual, 9: 59-66.

[4] Astrom, K. J., and Haggl and, T. 1995.“PID controllers: Theory, Design and Tuning”, Instr Soc. America, North Carolina.

[5] Yu, C. C. 1999. "Auto Tuning of PID controllers", Springer-Verlag, Berlin

[6] Ziegler, J. G. and Nichols, N. B. 1942.Optimum settings for automatic controllers. Trans ASME, 64: 759-765.

[7] Tyreus, B.D. and Luyben, W.L. 1992.Tuning PI controllers for integrator/dead-time processes. IndEngChem Res,31: 2625.

[ 8] Chidambaram, M. 1994. Design of PIcontroller for integrator/dead-time processes.Hungar J IndChem, 22: 37.

[ 9] Poulin, E. and Pomerleau, A. 1999. PIsettings for integrating processes basedon ultimate cycle information. IEEE Trans ContrSyst Tech, 7: 509.

[10] Luyben, W. L. 1996. Design of ProportionalIntegral and Derivative controllers for integrating dead-time processes. IndEngChem Res, 35: 3480.

[11] Wang, L. and Cluette, W.R. 1997. Tuning PID controllers for integrating processes.IEE ProcContrTheorAppl, 144: 385.

[12] Kookos, I. K., Lygros, A. I., and Arvanitis,K. G. 1999. On-line PI controller tuningfor integrator / dead time processes.Eur J Contr, 5: 19.

[13] Wang, Y.G. and Cai, W.J. 2002. Advanced proportional integral derivative tuning for integrating and unstable processes with gain and phase margin specifications .IndEngChem Res, 41:2910-2914.

[14] Visioli, A. 2001. Optimal tuning of PID controllers for integral and unstable processes. IEE ProcContrTheorAppl,148: 180.

[15] Zhang, W., Xu, X., and Sun, Y. 1999.Quantitative performance design for integrating processes with time delay. Automatica, 35: 719-723.

[16] Ali, A. and Majhi, S. 2010. PID controller tuning for integrating processes. ISA Trans, 49: 70-78.

[17] Rivera, D.E., Morari, M., and Skogestad, S. 1986. Internal Model Control - 4, PIDcontroller design. IndEngChem Process Des Dev, 25: 1684. 
[18] Skogestad, S. 2003. Simple analytical rules for model reduction and PID controllertuning. J Process Contr, 13: 291-309.

[19] Shamsuzzoha, Md. and Lee, M. 2008. PID controller design for integrating processes with time delay. Kor $J$ Chem Eng, 25, 4: 637-645.
[20] Sung, S.W. and Lee, I.B. 1996. Limitations and counter measures of PID controllers. Ind Eng Chem Res, 35: 2596-2610. 